\title{
Salient Signature of van der Waals Interactions
}

\author{
Mireia Via-Nadal, ${ }^{1}$ Mauricio Rodríguez-Mayorga, ${ }^{1,2}$ and Eduard Matito ${ }^{1,3}$ * \\ ${ }^{1}$ Kimika Fakultatea, Euskal Herriko Unibertsitatea (UPV/EHU), \\ and Donostia International Physics Center (DIPC), P.K. 1072, 20080 Donostia, Euskadi, Spain. \\ ${ }^{2}$ Institut de Química Computacional i Catàlisi (IQCC) and Departament de Química, \\ University of Girona, 17071 Girona, Catalonia, Spain, and \\ ${ }^{3}$ IKERBASQUE, Basque Foundation for Science, 48011 Bilbao, Euskadi, Spain.
}

(Dated: November 15, 2017)

\begin{abstract}
van der Waals interactions govern the physics of a plethora of molecular structures. It is well known that the leading term in the distance-based London expansion of the van der Waals energy for atomic and molecular dimers decays as $1 / R^{6}$, where $R$ is the dimer distance. Using perturbation theory, we find the leading term in the distance-based expansion of the intracule pair density at the interatomic distance. Our results unveil a universal $1 / R^{3}$ decay, which is less prone to numerical errors than the $1 / R^{6}$ dependency, and it is confirmed numerically in $\mathrm{H}_{2}$ and $\mathrm{He}_{2}$ molecules. This signature of van der Waals interactions can be directly used in the construction of approximate pair density and energy functionals including vdW corrections.
\end{abstract}

Dispersion or van der Waals (vdW) interactions are ubiquitous in nature, governing the stability of molecules and materials, $\frac{1}{,}$ and having an essential role in molecular recognition, ${ }^{2}$ the double-helical structure of $\mathrm{DNA}^{,}, \frac{3}{3}$ molecular adsorption on surfaces,,$\frac{4,5}{5}$ and the adhesion of micromachined surfaces $\underline{6}$ They are so important in physics, chemistry and biology, that even the most simple electronic structure methods consider corrections for vdW interactions. Due to their long-range dynamiccorrelation nature, they are not well modelled by standard functionals in density functional theory (DFT),$\frac{1}{2}$ which by construction are essentially local or semi-local in nature ${ }^{7}$ Hence, except for a few functionals, $\stackrel{8,9}{m}$ most DFT functionals include ad hoc energy corrections to account for vdW interactions $10-12$

vdW forces arise from the electrostatic interaction between fluctuations in the electron density, and the pairwise effect in the energy shows a leading $1 / R^{6}$ dependency, where $R$ is the interaction distance between fragments. This fact is often exploited in the construction of effective pairwise potentials that enter the expressions of various methods. On the other hand, the effect of vdW interactions in the wavefunction or related quantities has been less discussed in the literature ${ }^{13-16}$ This knowledge could shed some light in the design of computational approaches including vdW interactions and provide further tests to calibrate electronic structure theory methods.

In this letter we use perturbation theory to find the leading term in the expansion of the intracule pair density in terms of $R$, the interatomic distance. Our results reveal a universal $1 / R^{3}$ dependency that is corroborated by numerical calculations in $\mathrm{H}_{2}$ and $\mathrm{He}_{2}$ molecules. Upon integration of the vdW contribution to the intracule we recover the vdW energy that follows the stablished $1 / R^{6}$ dependency.

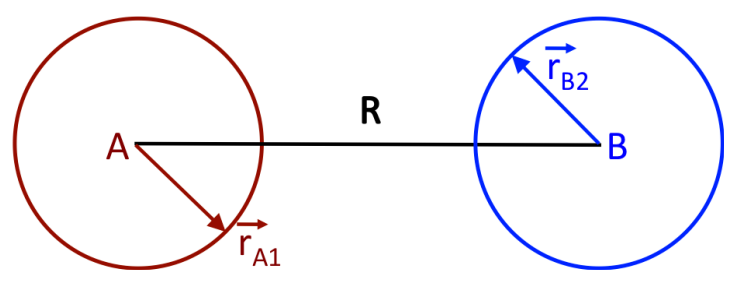

FIG. 1. Two atoms, $A$ and $B$, separated by a distance $R$ and two electrons with coordinates $\vec{r}_{A 1}$ and $\vec{r}_{B 2}$ defined with respect to the position of atoms $A$ and $B$.

\section{THEORY}

We start from the unperturbed wavefunction for a system of two hydrogen atoms, $A$ and $B$, separated a distance $R$, given by the product of two hydrogenoid $1 \mathrm{~s}$ functions 17

$$
\Psi^{(0)}\left(\mathbf{r}_{1}, \mathbf{r}_{2}\right)=N \phi_{A}\left(\mathbf{r}_{1}\right) \phi_{B}\left(\mathbf{r}_{2}\right) .
$$

For convenience we have chosen to represent the hydrogenoid functions by one Gaussian function of exponent $\alpha$. This change does not alter the $1 / R^{6}$ dependency on the calculation of the second-order correction to the energy (vide infra).

The perturbation operator contains all the interactions between fragments $A$ and $B$ :

$$
\hat{H}^{(1)}=-\frac{1}{r_{A 2}}-\frac{1}{r_{B 1}}+\frac{1}{R}+\frac{1}{r_{12}},
$$

where we have labeled the electrons in $A$ and $B$ as 1 and 2, respectively (see Fig. 1). After application of Unsöld's approximation, $\stackrel{18,19}{2}$ the expansion of Eq. 2 in terms of $1 / R$ at large distances yields the first-order correction to the wavefunction,

$$
\begin{aligned}
\Psi^{(1)}\left(\mathbf{r}_{1}, \mathbf{r}_{2}\right) & \approx \frac{\Psi^{(0)}\left(\mathbf{r}_{1}, \mathbf{r}_{2}\right)}{R^{3}}\left(x_{A 1} x_{B 2}+y_{A 1} y_{B 2}-2 z_{A 1} z_{B 2}\right) \\
& +\mathcal{O}\left(R^{-4}\right)
\end{aligned}
$$


where we have assumed that the molecule is located in the z-axis and $x_{A 1}, y_{B 2}$, etc. refer to the Cartesian components of the vectors in Eq. 2 Using the first-order correction to the wavefunction we can demonstrate that the leading term in the second-order correction to the energy follows the widely known $1 / R^{6}$ dependency $\underline{17}$

$$
E^{(2)}=\frac{6}{\alpha^{4} R^{6}}+\mathcal{O}\left(R^{-8}\right) .
$$

Now, let us consider the first-order correction to the pair density,

$$
n_{2}^{(1)}\left(\mathbf{r}_{1}, \mathbf{r}_{2}\right)=2 \Psi^{(0)}\left(\mathbf{r}_{1}, \mathbf{r}_{2}\right) \Psi^{(1)}\left(\mathbf{r}_{1}, \mathbf{r}_{2}\right) .
$$

The intracule of the pair density is a function that provides a distribution of the interelectronic separations, 20

$$
I(u)=\iint d \mathbf{r}_{1} d \mathbf{r}_{2} n_{2}\left(\mathbf{r}_{1}, \mathbf{r}_{2}\right) \delta\left(u-r_{12}\right),
$$

and thus returns the average electron-electron distance upon integration over $u$. The intracule of the pair density is actually connected with an experimental observable, the X-ray scattering intensity, which is essentially determined by the Fourier-Bessel transform of the radial intracule probability density: 21,22

After some algebraic manipulation one can prove that the zeroth-order intracule of the pair density at $R$,

$$
I^{(0)}(R)=\left(\frac{\alpha}{16 \pi^{3}}\right)^{1 / 2}\left(1-e^{-4 \alpha R^{2}}\right)
$$

yields a constant value in the limit, which corresponds to the distribution of two independent electrons. A Gaussian function enters the expression in Eq. 7 and, therefore, the form of the zeroth-order intracule function at $R$ depends on the reference wavefunction, Eq. 1. Conversely, the first-order correction at $R$ decays as $1 / R^{3}$,

$$
\lim _{R \rightarrow \infty} I^{(1)}(R)=-\frac{4(1+8 \sqrt{2}) \alpha^{5 / 2}}{\pi^{7 / 2} R^{3}},
$$

without any exponential terms in the expression, suggesting that the $1 / R^{3}$ dependency does not rely on a particular choice of the zeroth-order reference. As we will check numerically in the next section, Eq. 8 puts forward a universal condition that can be employed to assess the performance of approximate pair densities and models of the intracule function in reproducing vdW interactions.

\section{NUMERICAL EXAMPLES}

As a zeroth-order pair density we choose

$$
n_{2}^{\mathrm{SD}}\left(\mathbf{r}_{1}, \mathbf{r}_{2}\right)=n\left(\mathbf{r}_{1}\right) n\left(\mathbf{r}_{2}\right)-n_{1}\left(\mathbf{r}_{1} ; \mathbf{r}_{2}\right) n_{1}\left(\mathbf{r}_{2} ; \mathbf{r}_{1}\right),
$$

where $n_{1}\left(\mathbf{r}_{1} ; \mathbf{r}_{2}\right)$ is the first-order reduced density matrix. $n_{2}^{\mathrm{SD}}$ is the minimal model that guarantees that $n_{2}^{\mathrm{SD}}\left(\mathbf{r}_{1}, \mathbf{r}_{2}\right) \rightarrow n\left(\mathbf{r}_{1}\right) n\left(\mathbf{r}_{2}\right)$ at large interatomic distances and, at the same time, preserves the antisymmetric nature of particles, ${ }^{23}$ which is necessary to remove the spin entanglement effects that also appear at large interelectronic separations and are not included by the secondorder perturbational treatment. Hence, we will evaluate the intracule resulting from the following pair density difference

$I^{(1)}(u) \approx \iint d \mathbf{r}_{1} d \mathbf{r}_{2}\left[n_{2}\left(\mathbf{r}_{1}, \mathbf{r}_{2}\right)-n_{2}^{\mathrm{SD}}\left(\mathbf{r}_{1}, \mathbf{r}_{2}\right)\right] \delta\left(u-r_{12}\right)$

To this aim, we have chosen two simple molecules, $\mathrm{H}_{2}$ and $\mathrm{He}_{2}$, and we have performed full-configuration interaction calculations 24 with the aug-cc-pVDZ basis set at different interatomic separations $(R)$. We have computed Eq. 10 and plotted $I^{(1)}(R)$ against $R$ (see Fig. 2).

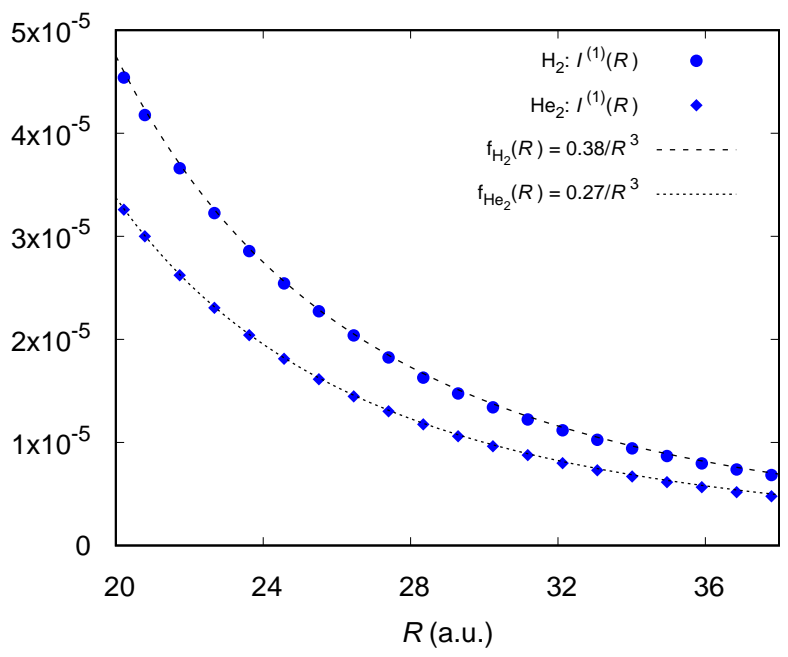

FIG. 2. $I^{(1)}(R)$ against $R$, the interatomic distance, for $\mathrm{H}_{2}$ and $\mathrm{He}_{2}$. The fitting corresponds to $f(R)=a / R^{3}$, where $a$ is the fitted parameter and equals 0.38 and 0.27 for $\mathrm{H}_{2}$ and $\mathrm{He}_{2}$, respectively. In both cases the Pearson regression coefficient, $r^{2}$, is greater than 0.999. All quantities are in a.u.

The numerical results confirm the predicted $1 / R^{3}$ dependency of the vdW correction to the intracule of the pair density. The fitting procedure employs the points presented in Fig. 2 and uses a least squares minimization analysis to determine the Pearson regression coefficient and the $a$ parameter in the $f(R)=a / R^{3}$ fitting function.

The intracule pair density is the simplest quantity in terms of which we can express explicitly and exactly the electron-electron repulsion energy,

$$
V_{e e}=\int d u \frac{I(u)}{u}
$$

and the integration of the first-order correction to the intracule divided by the electron-electron distance provides the vdW energy:

$$
V_{e e}^{(2)}=\int d u \frac{I^{(1)}(u)}{u} .
$$


Through Eq. 12, we can calculate the vdW energy using the first-order correction to the intracule function. In Fig. 3 we observe that at large distances these functions reproduce the $1 / R^{6}$ dependency. The fitting is not as good as in the intracule function because of the large power dependency, which increases the numerical error.

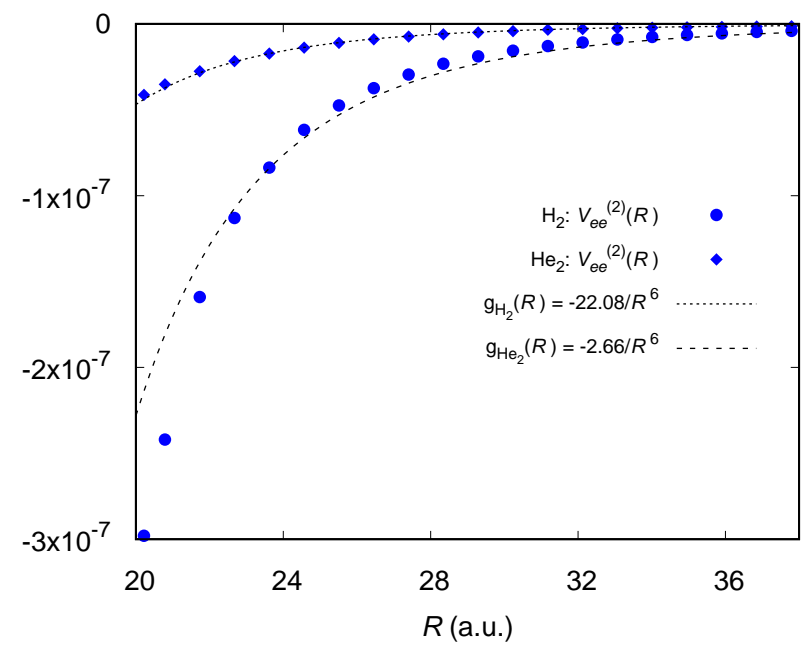

FIG. 3. $V_{e e}^{(2)}(R)$ against $R$, the interatomic distance, for $\mathrm{H}_{2}$ and $\mathrm{He}_{2}$. The fitting corresponds to $g(R)=a / R^{6}$, where $a$ is the fitted parameter and equals -22.08 and -2.66 for $\mathrm{H}_{2}$ and $\mathrm{He}_{2}$, respectively. In both cases the Pearson coefficient, $r^{2}$, is greater than 0.97. All quantities are in a.u.

\section{CONCLUSIONS}

We have unveiled a universal condition of the intracule of the pair density: the vdW contribution to the intracule of the pair density at $R$ should decay as $1 / R^{3}, R$ being the separation of two fragments. This condition is connected to the well-known $1 / R^{6}$ decay of the vdW energy and it can be recovered from the vdW contribution to the intracule of the pair density (see Eq. 12). This requirement is a salient signature of vdW interactions that can be employed as a stringent constraint in a judicious construction of new methods and approximations in electronic structure theory including vdW interactions ${ }^{25-27}$ Since the vdW correction to the intracule of the pair density shows a lower power dependency than the energy one, it is also less prone to numerical errors (compare fittings in Figs. 2 and 3 ).

\section{ACKNOWLEDGMENTS}

The authors thank Dr. Eloy Ramos-Cordoba for helpful discussions. This research has been funded by Spanish MINECO/FEDER Project CTQ2014-52525-P and the Basque Country Consolidated Group Project No. IT588-13. M.V.N. and M.R.M. acknowledge the Spanish Ministry of Economy, Industry and Competitiveness (MINECO) and the Spanish Ministry of Education, Culture and Sports for the doctoral grants BES-2015-072734 and FPU-2013/00176, respectively.
* To whom the correspondence should be addressed: ematito@gmail.com

1 J. Hermann, R. A. DiStasio Jr, and A. Tkatchenko, Chem. Rev. 117, 4714 (2017).

2 L. Yang, C. Adam, G. S. Nichol, and S. L. Cockroft, Nat. Chem. 5, 1006 (2013).

3 J. Černý, M. Kabelác, and P. Hobza, J. Am. Chem. Soc. 130, 16055 (2008).

4 J. Klimeš, D. R. Bowler, and A. Michaelides, Phys. Rev. B 83, 195131 (2011).

5 M. Rosa, S. Corni, and R. Di Felice, Phys. Rev. B 90, 125448 (2014)

${ }^{6}$ F. W. DelRio, M. P. de Boer, J. A. Knapp, E. D. Reedy, P. J. Clews, and M. L. Dunn, Nat. Mat. 4, 629 (2005).

7 S. Kristyán and P. Pulay, Chem. Phys. Lett. 229, 175 (1994).

8 K. Berland, V. R. Cooper, K. Lee, E. Schröder, T. Thonhauser, P. Hyldgaard, and B. I. Lundqvist, Rep. Prog. Phys. 78, 066501 (2015).

9 T. Thonhauser, V. R. Cooper, S. Li, A. Puzder, P. Hyldgaard, and D. C. Langreth, Phys. Rev. B 76, 125112 (2007)

10 S. Grimme, WIREs, Comput. Mol. Sci. 1, 211 (2011).

11 A. D. Becke and E. R. Johnson, J. Chem. Phys. 123, 154101 (2005)
12 A. D. Becke and E. R. Johnson, J. Chem. Phys. 127, 154108 (2007).

${ }^{13}$ K. Rapcewicz and N. Ashcroft, Phys. Rev. B 44, 4032 (1991).

14 P. Hyldgaard, K. Berland, and E. Schröder, Phys. Rev. B 90, 075148 (2014).

15 N. Ferri, R. A. DiStasio Jr, A. Ambrosetti, R. Car, and A. Tkatchenko, Phys. Rev. Lett. 114, 176802 (2015).

16 N. Ferri, A. Ambrosetti, and A. Tkatchenko, Phys. Rev. Mat. 1, 026003 (2017)

17 L. Pauling and E. B. Wilson, Introduction to quantum mechanics (Dover Publications, Inc., New York, 1935).

18 A. Unsöld, Z. Physik 43, 563 (1927).

19 J. O. Hirschfelder, W. B. Brown, and S. T. Epstein, Adv. Quantum Chem. 1, 255 (1964).

20 C. A. Coulson and A. H. Neilson, Proc. Phys. Soc. London 78, 831 (1961).

21 A. J. Thakkar, A. Tripathi, and V. H. Smith, Int. J. Quant. Chem. 26, 157 (1984).

22 A. J. Thakkar, A. Tripathi, and V. H. Smith Jr, Phys. Rev. A 29, 1108 (1984).

23 M. Rodríguez-Mayorga, E. Ramos-Cordoba, M. ViaNadal, M. Piris, and E. Matito, Phys. Chem. Chem. Phys. 19, 24029 (2017).

24 The calculations have been performed with a modified version of the program of Knowles and Handy ${ }^{28}$ and the pair 
density matrices have been obtained with our in-house DMN code ${ }^{29}$ The intracule calculation employed the inhouse RHO2_OPS 30 code, which uses the algorithm ${ }^{31}$ of Cioslowski and Liu.

25 D. A. Mazziotti, Phys. Rev. Lett. 108, 263002 (2012).

26 A. W. Schlimgen, C. W. Heaps, and D. A. Mazziotti, J. Phys. Chem. Lett. 7, 627 (2016).

27 D. A. Mazziotti, Phys. Rev. Lett. 117, 153001 (2016).
28 P. J. Knowles and N. C. Handy, Comput. Phys. Commun. 54, 75 (1989).

29 E. Matito and F. Feixas, "DMn program," (2009), university of Girona (Spain) and University of Szczecin (Poland).

30 M. Rodríguez-Mayorga, "RHO2-OPS: 2-dm operations." (2016), institute of Computational Chemistry and Catalysis, University of Girona, Catalonia, Spain.

31 J. Cioslowski and G. Liu, J. Chem. Phys. 105, 4151 (1996). 